\title{
Needs, priorities, and attitudes of individuals with spinal cord injury toward nerve stimulation devices for bladder and bowel function:
}

\section{a survey}

\author{
Dennis Bourbeau $\mathbb{1}^{1,2,3} \cdot$ Abby Bolon $^{4} \cdot$ Graham Creasey $^{5} \cdot$ Wei Dai $^{4} \cdot$ Bill Fertig $^{6} \cdot$ Jennifer French $^{7} \cdot$ Tara Jeji $^{8}$. \\ Anita Kaiser $\mathbb{1}^{9,10} \cdot$ Roman Kouznetsov ${ }^{4}$. Alexander Rabchevsky ${ }^{11} \cdot$ Bruno Gallo Santacruz $\mathbb{D}^{12} \cdot$ Jiayang Sun $^{13}$. \\ Karl B. Thor ${ }^{14} \cdot$ Tracey Wheeler $^{15} \cdot$ Jane Wierbicky ${ }^{16}$
}

Received: 21 December 2019 / Revised: 21 August 2020 / Accepted: 26 August 2020 / Published online: 7 September 2020

This is a U.S. government work and not under copyright protection in the U.S.; foreign copyright protection may apply 2020

\begin{abstract}
Study design Survey.

Objectives To investigate the needs and priorities of people with spinal cord injury for managing neurogenic bladder and bowel function and to determine their willingness to adopt neuromodulation interventions for these functions.

Methods Anonymous online survey. It was advertised by word-of-mouth by community influencers and social media, and by advertisement in newsletters of advocacy groups.

Results Responses from 370 individuals ( $27 \%$ female, $73 \%$ male) were included. Bladder emptying without catheters was the top priority for restoring bladder function, and maintaining fecal continence was the top priority for restoring bowel function. The biggest concerns regarding external stimulation systems were wearing a device with wires connecting to electrodes on the skin and having to don and doff the system daily as needed. The biggest concerns for implanted systems were the chances of experiencing problems with the implant that required a revision surgery or surgical removal of the whole system. Respondents were willing to accept an external (61\%) or implanted (41\%) device to achieve improved bladder or bowel function.

Conclusions Bladder and bowel dysfunction remain important unmet challenges for individuals living with SCI who answered our survey. These individuals are willing to accept some potential risks of nerve stimulation approaches given potential benefits. Additional consumer input is critical for guiding both research and translation to clinical use and personalized medicine.
\end{abstract}

Supplementary information The online version of this article (https:// doi.org/10.1038/s41393-020-00545-w) contains supplementary material, which is available to authorized users.

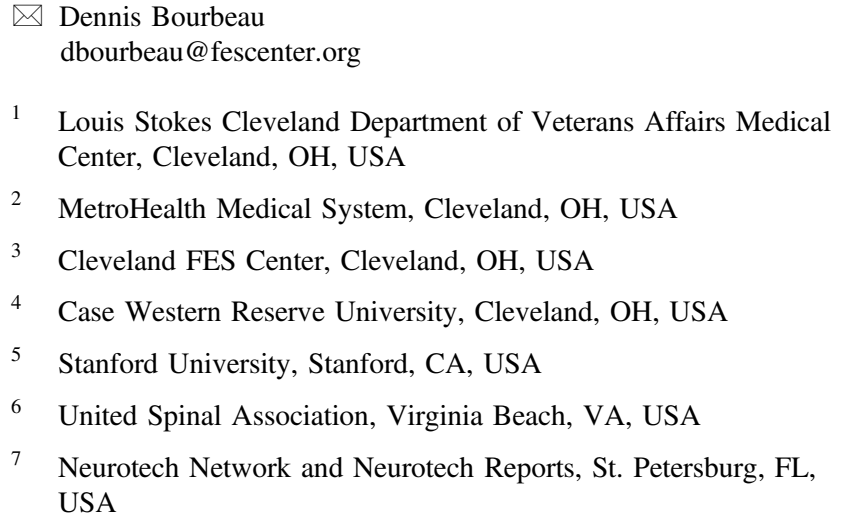
USA

\section{Introduction}

Virtually all individuals living with spinal cord injury (SCI) experience neurogenic bladder and/or bowel dysfunction,

\footnotetext{
8 Ontario Neurotrauma Foundation, Toronto, ON, Canada

9 Toronto Rehabilitation Institute-University Health Network, Toronto, ON, Canada

10 Canadian Spinal Research Organization, Toronto, ON, Canada

11 SCoBIRC, University of Kentucky, Lexington, KY, USA

12 Radiometer, Copenhagen, Denmark

13 Department of Statistics, George Mason University, Fairfax, VA, USA

14 Dignify Therapeutics Inc., Durham, NC, USA

15 Craig H. Neilsen Foundation, Encino, CA, USA

16 United Spinal Association, Kew Gardens, NY, USA
} 
which can have significant adverse impacts on health, independence, self-image, and overall quality of life. Restoration of bladder and bowel functions are high priorities as identified by people living with paraplegia and quadriplegia [1-3]. The inability to control elimination of urine and feces are persistent issues for people living with SCI and can reduce participation in relationships, activities, and employment options, as well as impede dignity and privacy $[4,5]$. The importance of neurogenic bladder and bowel dysfunction has been acknowledged generally, but stakeholders, including healthcare professionals, researchers, industry partners, regulatory agencies, funding agencies, and advocacy groups, would benefit from a deeper understanding of the specific bladder and bowel needs and priorities of individuals with SCI. This specificity would help define more specific targets for restoration of bladder and bowel function.

Existing and emerging approaches that use stimulation of the nerves innervating the pelvic organs have the potential to improve or restore bladder and bowel functions following SCI [6]. One approach, sacral anterior root stimulation, has been provided to thousands of individuals with SCI to promote bladder and bowel emptying [7-9]. Another approach using stimulation of sensory nerves can improve urinary continence in individuals with SCI [10-13]. There are also approaches that are in research and efforts are being made to translate them for human testing [14, 15]. Two of the key challenges for advancing these engineered neurostimulation approaches rests in demonstrating that they meet an important need as defined by individuals with SCI and that those individuals would be willing to adopt these solutions. To address these challenges, the literature provides important insights into the general needs of individuals with SCI, but further specificity on the needs and priorities of individuals with SCI is needed. There is also a need to ascertain the potential interest of individuals with SCI in neurostimulation approaches, which could drive the direction of technology advancement [16]. This need for end-user input and further study of it has been recognized and strongly recommended [17].

The purpose of this study was to provide preliminary assessment of (1) the needs and priorities of individuals living with SCI for improving or restoring their bladder and bowel functions to provide context for (2) their willingness to adopt nerve stimulation approaches to improve or restore these functions.

\section{Methods}

We formed a working group, including nine individuals from research, industry, non-profit, and clinical backgrounds. The working group collaborated with the North American Spinal Cord Injury Consortium (NASCIC) to assemble a consumer advisory board. The consumer advisory board consisted of five individuals living with SCI, including women and men with various levels of injury and durations since injury, and one registered nurse with expertise in SCI. This group provided a person-centered perspective to meet study objectives.

Together these two groups developed a Voice of Customer survey tool. This survey tool was pilot tested by five individuals living with SCI, who were randomly selected by NASCIC, and we revised questions based on this test feedback. The survey was then disseminated using SurveyMonkey Inc. (https://www.surveymonkey.com) and was advertised via standard communication channels, such as newsletters, advocacy groups representing the SCI community, social media, and by word-of-mouth. This methodology may introduce different sources of bias (see "Study limitations"), but the survey was designed to explore perspectives of individuals living with SCI. The full survey is provided as a supplement to this manuscript. Individuals completed this online survey between 28 June and 29 August 2018.

\section{Measurements and analysis}

Questions were sorted into four categories: demographics, bladder function, bowel function, and attitudes toward nerve stimulation. Questions regarding bladder and bowel function and management were separated into two respective sections, but the questions were similar for both. These questions included methods and challenges of managing their bladder or bowel function; how much their bladder or bowel management interferes with their daily routines, professional activities, and social activities; and how they rank the bladder and bowel functions that they would like to have improved or restored. These forced rank questions directly asked respondents about their priorities for improving or restoring bladder and bowel function and was a main outcome measure for the study.

The survey asked about respondents' willingness to accept potential risks related to nerve stimulation approaches, including external and implanted systems, when considering potential benefits of improved or restored function. We presented a general definition of a nerve stimulation device, including potential external and implanted versions, to inform the respondents before asking them about their willingness to accept these devices to improve or restore bladder and bowel functions. We presented known risks with estimated rates of occurrence based on the literature for similar technologies [18-20]. We asked respondents to rate their level of concern for each of these risks. The respondents' willingness to accept external or implanted nerve stimulation devices to improve or restore bladder and bowel functions was a main outcome measure for the study. 
Survey data were analyzed using MATLAB R2018a (MathWorks Inc., Natick, MA) and R Programming Language (Version 3.0.2; R Foundation for Statistical Computing, Vienna, Austria). Informative summary statistics were provided numerically and graphically.

\section{Results}

\section{Demographics}

Data from 370 survey respondents were included in this analysis because they reported that they were over 18 years of age, and they confirmed that they are living with SCI (see Table 1 for demographics summary). Three hundred nineteen of those respondents live in the United States. Respondents tended to consult urologists and primary care physicians the most to help manage their bladder and bowel concerns. Their healthcare providers and the internet were frequently sought for information regarding their bladder and bowel management. Friends, peer support groups, and advocacy organizations were also important sources for information.

\section{Bladder function}

Many respondents have frequent need for bladder emptying; need for assistance with bladder management; and difficulty sensing when their bladder is full (Table 2). Ninety-four percent of respondents required some form of bladder management method and $25 \%$ of respondents used more than one method. Eighty-six percent of respondents reported using various types of catheterization methods. Approximately half of respondents use medications to help manage their bladder. Only six respondents reported use of electrical stimulation as part of their bladder management strategy. In the past 12 months, most respondents experienced challenges associated with their bladder management, and 55\% experienced two or more of these challenges. Urinary tract infections followed by episodes of urinary incontinence were the most common complications reported by respondents. Approximately $70 \%$ of respondents found that their bladder at least somewhat interfered with daily, work, and social activities (Fig. 1a). When asked to rank priorities for restoring function, we found that emptying their bladder without catheters was the number one priority, followed by maintaining urinary continence (Fig. 1b).

\section{Bowel function}

Bowel management data summarized in Table 3 suggest that respondents typically require a long time to empty their bowels; require assistance for their bowel management; and
Table 1 Respondent demographics.

\begin{tabular}{|c|c|c|}
\hline Age & 50 (22) years & \\
\hline Age at Injury & 29 (24) years & \\
\hline Years since SCI & $12(22)$ years & \\
\hline \multicolumn{3}{|l|}{ Sex } \\
\hline Women & $27 \%$ & $N=100$ \\
\hline Men & $73 \%$ & $N=269$ \\
\hline \multicolumn{3}{|l|}{ Hand function } \\
\hline No hand function & $15 \%$ & $N=55$ \\
\hline Some hand function & $28 \%$ & $N=104$ \\
\hline Full hand function & $57 \%$ & $N=211$ \\
\hline \multicolumn{3}{|l|}{ Performing transfers } \\
\hline Full assistance & $18 \%$ & $N=66$ \\
\hline Some assistance & $22 \%$ & $N=83$ \\
\hline No assistance & $59 \%$ & $N=219$ \\
\hline \multicolumn{3}{|l|}{ Leaving the house } \\
\hline Never & $2 \%$ & $N=8$ \\
\hline Monthly & $7 \%$ & $N=25$ \\
\hline Weekly & $26 \%$ & $N=98$ \\
\hline Daily & $64 \%$ & $N=237$ \\
\hline \multicolumn{3}{|l|}{ Transportation barriers } \\
\hline Yes & $13 \%$ & $N=49$ \\
\hline No & $86 \%$ & $N=319$ \\
\hline \multicolumn{3}{|l|}{ Financial barriers } \\
\hline Yes & $25 \%$ & $N=93$ \\
\hline No & $75 \%$ & $N=277$ \\
\hline \multicolumn{3}{|c|}{ Healthcare professionals consulted } \\
\hline Urologist & $70 \%$ & $N=259$ \\
\hline Primary care physician & $60 \%$ & $N=221$ \\
\hline Nurse & $22 \%$ & $N=80$ \\
\hline Physiatrist & $21 \%$ & $N=77$ \\
\hline Neurologist & $12 \%$ & $N=46$ \\
\hline Gastroenterologist & $12 \%$ & $N=44$ \\
\hline Neurosurgeon & $4 \%$ & $N=13$ \\
\hline Free clinic & $0.5 \%$ & $N=2$ \\
\hline Other & $7 \%$ & $N=27$ \\
\hline No consultation & $2 \%$ & $N=7$ \\
\hline \multicolumn{3}{|c|}{ Sources of healthcare information } \\
\hline Medical providers & $82 \%$ & $N=305$ \\
\hline Internet & $64 \%$ & $N=238$ \\
\hline Friends and family & $27 \%$ & $N=99$ \\
\hline Peers & $26 \%$ & $N=97$ \\
\hline Advocacy groups & $23 \%$ & $N=84$ \\
\hline Information sessions & $12 \%$ & $N=44$ \\
\hline Television & $4 \%$ & $N=14$ \\
\hline Newspaper & $2 \%$ & $N=6$ \\
\hline Other & $8 \%$ & $N=28$ \\
\hline
\end{tabular}

have difficulty sensing when their bowel is full, which makes it challenging to determine when to empty in order to avoid 
Table 2 Bladder management.

\section{Bladder emptying frequency}

Not applicable-I use a drainage bag or constant

draining system

1-2 times/day

$3-4$ times/day

5-6 times/day

More than 6 times/day

Bladder medications

Yes

No

I do not know

Assistance with bladder

Full assistance

Some assistance

No assistance

Assistance if incontinent

Full assistance

Some assistance

No assistance

No incontinence

Sense when bladder is full

Not applicable_constant drain

Yes

No

If bladder sense, avoid incontinence

Yes

No

Bladder management methods

Clean intermittent catheterization

Medication

Absorbent pads or diapers

Suprapubic catheter

Condom catheter

Indwelling or foley catheter

Electrical stimulation

Other

Challenges associated with bladder management in last 12 months

Urinary tract infection

Urinary incontinence

Autonomic dysreflexia

Clogged catheter

Bladder or kidney stones

Kidney disease or failure

Other
$21 \% \quad N=76$
$4 \% \quad N=15$
$22 \% \quad N=83$
$38 \% \quad N=141$
$14 \% \quad N=51$

$48 \% \quad N=176$

$50 \% \quad N=184$

$1 \% \quad N=5$

$13 \% N=49$

$13 \% N=48$

$72 \% \quad N=268$

$20 \% \quad N=73$

$15 \% \quad N=57$

$42 \% \quad N=156$

$21 \% N=79$

$13 \% N=48$

$60 \% \quad N=221$

$26 \% N=96$

$74 \% \quad N=163$

$23 \% N=50$

$59 \% \quad N=219$

$48 \% \quad N=176$

$21 \% \quad N=79$

$15 \% \quad N=57$

$15 \% \quad N=55$

$9 \% \quad N=33$

$2 \% \quad N=6$

$6 \% \quad N=24$

$65 \% \quad N=240$

$49 \% \quad N=182$

$30 \% \quad N=112$

$16 \% N=58$

$14 \% \quad N=50$

$2 \% \quad N=7$

$8 \% \quad N=31$

episodes of fecal incontinence. Ninety-six percent of respondents used some form of bowel management method and $64 \%$ used at least two methods. Sixty-five percent of respondents reported using digital rectal stimulation, $38 \%$ of

respondents reported using manual evacuation of stools, and $26 \%$ of respondents reported using both methods together. Sixty-two percent of respondents used suppositories, laxatives, and/or enemas. External adaptive devices, implanted devices, and colostomy bags were the least commonly used methods of bowel management. Forty-six percent of respondents used some combination of digital rectal stimulation or manual evacuation with suppositories, laxatives, or enemas.

Ninety-two percent of respondents reported experiencing at least some type of complication in the past 12 months due to their bowel management, and $70 \%$ reported multiple types of challenges. Constipation and fecal incontinence were the most frequently reported at $60 \%$ and $54 \%$, respectively. Episodes of autonomic dysreflexia (21\%) and colostomy complications $(2 \%)$ were the least frequently reported complications. As with the questions for bladder management, these data do not track the frequency with which these challenges occur. Respondents also reported similar interference with their everyday activities from their neurogenic bowel as from their neurogenic bladder (Fig. 2a). Maintaining fecal continence was the number one priority (Fig. 2b). Having predictability in the bowel routine and reducing the time required for the bowel routine were also ranked highly.

\section{Attitudes toward nerve stimulation approaches}

The included pool of 370 respondents rated how likely they would be to accept an implanted or external neurostimulation device on a scale from 1 to 7 , with 1 being "very likely" and 7 being "not likely" (Fig. 3a, b). Implanted devices were scored 3.3 (0.2), with 41 (4)\% of respondents reporting that they were very likely to accept an implanted device. External devices were considered more acceptable, receiving a score of $2.5(0.2)$, with $61(5) \%$ of respondents reporting that they were very likely to accept an external device. Respondents ranked in order, from greatest to least concern, the potential risks and inconveniences of implanted and of external neurostimulation devices. For implanted devices, the two biggest concerns were having a problem with the implant requiring a revision surgery, and problems with the implant requiring removal of the whole system (Fig. 3c). The two biggest concerns associated with using external devices were the inconvenience of wearing a device with wires connecting to electrodes under their clothes and having to don and doff the device daily or as needed (Fig. 3d).

\section{Discussion}

This report summarizes our findings from a Voice of Customer survey to collect information regarding the challenges with which individuals struggle and how they rank the 


\section{A: Interference from Bladder Management}

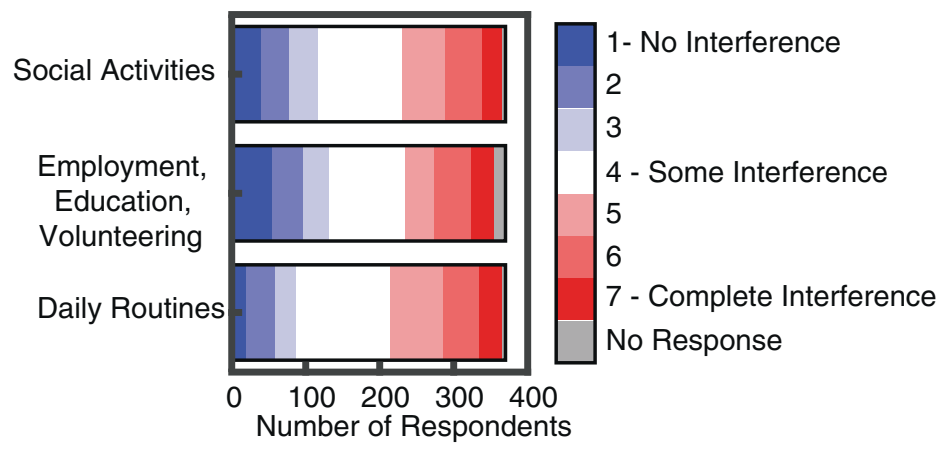

\section{B: Ranked Priorities for Bladder Function Restoration}
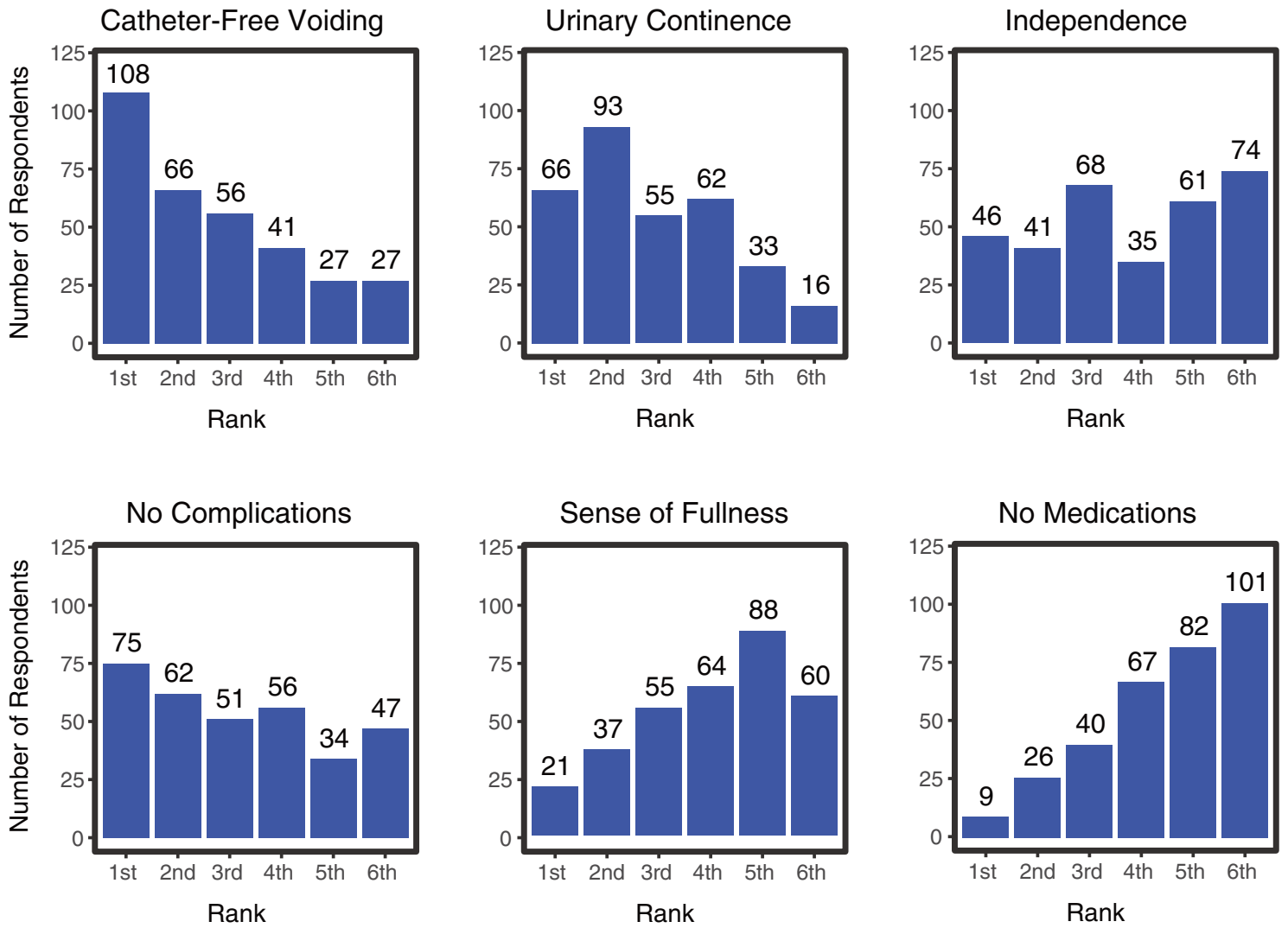

Fig. 1 Inconveniences from bladder management and priorities for functional improvements. a Respondents rated interference from bladder management on everyday activities on a scale from 1 to 7 , where 1 represents "no interference" and 7 represents "complete interference". Respondents reported that their bladder interfered with

importance of restoring specific bladder and bowel functions. We also present feedback on individuals' attitudes toward potential neurostimulation approaches to improve or restore these functions. The data presented in this report provide valuable insight that could help direct research efforts to improve or restore bladder and bowel function for individuals with SCI daily routines with an average score of 4.3 , with social activities at 4.0 , and with education, volunteering, and employment activities at 3.9. b Respondents responded to a forced-choice rank of six potential improvements in bladder function. For each bladder function priority, the distribution of the rank scores that it received are shown.

\section{Demographics}

We compared the distributions of age, sex, years since SCI, and age at SCI (see Table 1) in our data with those in the national database hosted by the National Spinal Cord Injury Statistical Center [21]. We found that these distributions match well with the distributions seen in the national 
Table 3 Bowel management.

\begin{tabular}{|c|c|c|}
\hline \multicolumn{3}{|l|}{ Bowel routine duration } \\
\hline More than $2 \mathrm{~h}$ & $5 \%$ & $N=20$ \\
\hline $1-2 \mathrm{~h}$ & $20 \%$ & $N=74$ \\
\hline $30-60 \min$ & $36 \%$ & $N=133$ \\
\hline$<30 \mathrm{~min}$ & $35 \%$ & $N=128$ \\
\hline NA-ostomy bag & $3 \%$ & $N=11$ \\
\hline \multicolumn{3}{|l|}{ Bowel routine frequency } \\
\hline Once/week & $3 \%$ & $N=12$ \\
\hline Twice/week & $9 \%$ & $N=34$ \\
\hline Thrice/week & $18 \%$ & $N=66$ \\
\hline Every other day & $23 \%$ & $N=84$ \\
\hline Daily & $42 \%$ & $N=157$ \\
\hline NA—ostomy bag & $3 \%$ & $N=11$ \\
\hline \multicolumn{3}{|l|}{ Daily bowel medications } \\
\hline Yes & $33 \%$ & $N=121$ \\
\hline No & $66 \%$ & $N=244$ \\
\hline I do not know & $1 \%$ & $N=2$ \\
\hline \multicolumn{3}{|l|}{ Assistance with bowel } \\
\hline Full assistance & $34 \%$ & $N=121$ \\
\hline Some assistance & $10 \%$ & $N=38$ \\
\hline No assistance & $56 \%$ & $N=209$ \\
\hline \multicolumn{3}{|c|}{ Assistance if incontinent of stool } \\
\hline Full assistance & $40 \%$ & $N=148$ \\
\hline Some assistance & $18 \%$ & $N=65$ \\
\hline No assistance & $31 \%$ & $N=114$ \\
\hline No incontinence & $11 \%$ & $N=42$ \\
\hline \multicolumn{3}{|l|}{ Sense when bowel is full } \\
\hline No & $48 \%$ & $N=176$ \\
\hline Yes & $48 \%$ & $N=179$ \\
\hline Not applicable—stoma & $4 \%$ & $N=14$ \\
\hline \multicolumn{3}{|l|}{ Bowel management methods } \\
\hline Digital rectal stimulation & $65 \%$ & $N=242$ \\
\hline Manual evacuation & $38 \%$ & $N=139$ \\
\hline Suppositories & $36 \%$ & $N=133$ \\
\hline Laxatives & $35 \%$ & $N=130$ \\
\hline Medications & $33 \%$ & $N=121$ \\
\hline Special diet & $18 \%$ & $N=65$ \\
\hline Enemas & $17 \%$ & $N=64$ \\
\hline Colostomy bag & $3 \%$ & $N=12$ \\
\hline External adaptive device & $2 \%$ & $N=7$ \\
\hline Implanted device & $1 \%$ & $N=5$ \\
\hline Other & $11 \%$ & $N=41$ \\
\hline \multicolumn{3}{|c|}{ Challenges associated with bowel management in last 12 months } \\
\hline Constipation & $60 \%$ & $N=222$ \\
\hline Fecal incontinence & $54 \%$ & $N=198$ \\
\hline Loose stools & $47 \%$ & $N=173$ \\
\hline Hemorrhoids & $45 \%$ & $N=167$ \\
\hline Bleeding & $39 \%$ & $N=143$ \\
\hline Autonomic dysreflexia & $21 \%$ & $N=76$ \\
\hline Colostomy complications & $2 \%$ & $N=8$ \\
\hline Other & $5 \%$ & $N=20$ \\
\hline
\end{tabular}

database. We also estimated the number of people that could have been reached with the various methods that we used, such as e-mail or online newsletter, etc. We could track and confirm response rates on two of those outreach methods and found those response rates to be 46 and $29 \%$. Our most conservative estimate suggests that our response rate was on average at least $1 \%$, which assumes that we reached out to $\sim 35,000$ individuals across outreach methods. This conservative estimate also assumes that there is no overlap between potential outreach populations and that everyone in that population has SCI; both of those assumptions are highly unlikely. Demographic data, including age, sex, and year since SCI, were not factors for missing data. That is, the percentage of missing data did not change as a function of these demographic factors, and missing data are highly likely attributed to the randomness in people who choose not to respond.

Urologists and primary care physicians were the most consulted health care professionals. It is not clear if these health care professionals had strong experience in SCI medicine. It is also not clear what impacts these rates of consultation may have on strategies for bladder and bowel management or access to appropriate information on best practices for spinal cord medicine. Expertise for bowel care, especially gastroenterologists, was not frequently sought. These rates could be due to perceived need or as a result of the structure of the healthcare system within which the respondent receives treatment. It should be noted that many respondents consulted with nurses regarding their bladder and bowel care, highlighting the importance of nursing in the delivery of healthcare. Healthcare providers and the internet were the two most popular sources of information, which was not unexpected, but social groups were also important. Advocacy groups and other individuals with SCI may provide practical information about different strategies for managing bladder and bowel function based on their lived experiences.

Respondents were not asked about their lesion level or completeness and instead were asked about their functional abilities relating to bladder and bowel function. The incidence and severity of neurogenic bladder and bowel depend on the level and grade of SCI. However, an individual's needs and priorities may be influenced by such things as hand function and the presence or absence of pelvic reflexes, whether or not they even know their SCI level and grade. While lesion level and completeness can provide insight into the likely bladder and bowel dysfunctions, we believe that it was more appropriate to directly ask about respondents' functions, such as hand function and the ability to transfer, which was communicated by consensus by our Consumer Advisory Board with SCI lived experience.

\section{Bladder management}

The majority of respondents did not require assistance with their bladder, which was consistent with the high rate of respondents reporting hand function and the ability to perform transfers without assistance. The high rates of 
A: Interference from Bowel Management

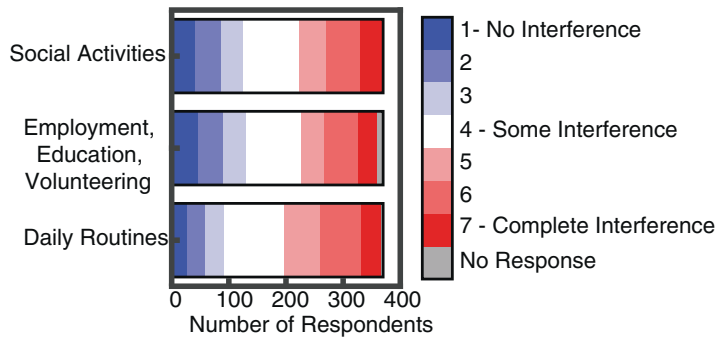

B: Ranked Priorities for Bowel Function Restoration
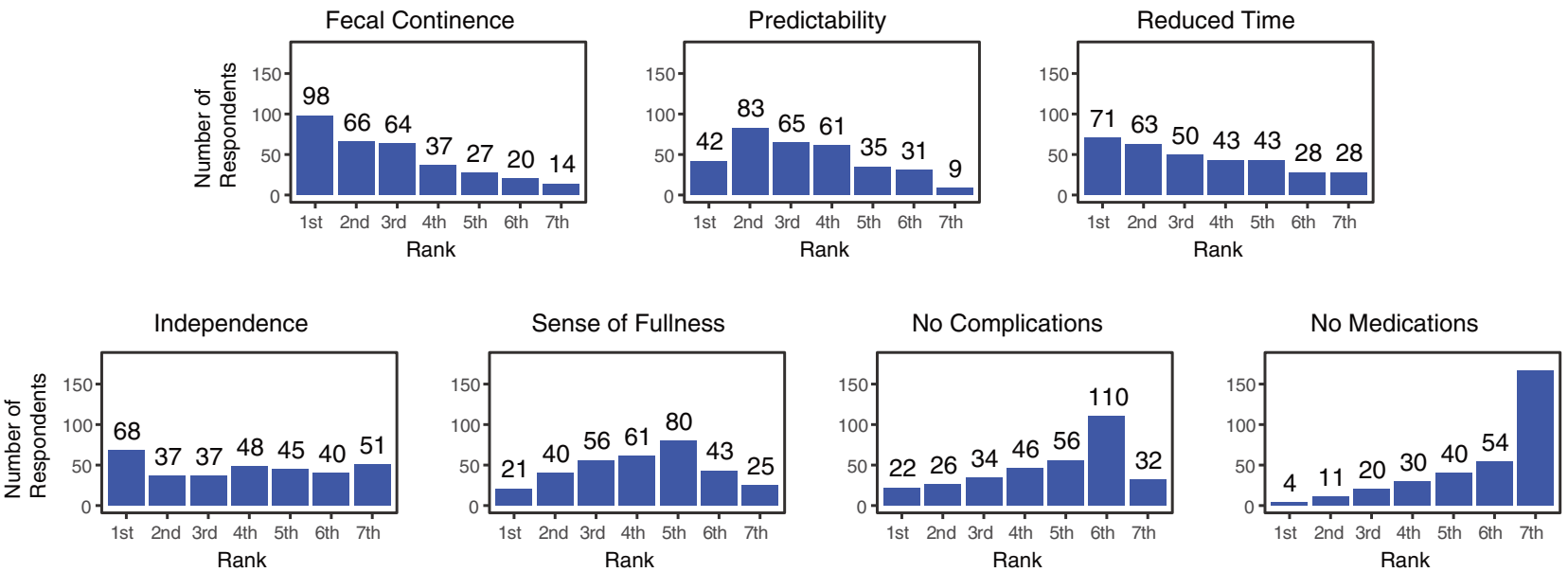

Fig. 2 Inconveniences from bowel management and priorities for functional improvements. a Respondents rated interference from bowel management on everyday activities on a scale from 1 to 7 , where 1 represents "no interference" and 7 represents "complete interference". Approximately $70 \%$ of respondents found that their

respondents reporting high bladder emptying frequencies; using bladder medications to help manage their bladder; and reporting at least one episode of urinary incontinence in the last year, suggest challenges from bladder overactivity and bladder urgency. Bladder management interfered with respondents' activities and many respondents reported issues with autonomic dysreflexia, bladder or kidney stones, or even kidney disease or kidney failure. It should be noted that our survey did not ask how many times these issues occurred in the past 12 months. Therefore, these data may understate the severity of these challenges. Thus, these data suggest that most individuals have some independence in managing their bladder function but struggle with the challenges and complications of urinary retention and/or bladder overactivity.

Emptying without catheters; being free of medical complications (e.g., urinary tract infections); and maintaining urinary continence were the highest ranked and very closely ranked, but respondents felt that all six issues were important. Prioritizing bladder emptying over urinary continence may be unexpected, but impaired bladder emptying bowel somewhat interfered with these activities. b Respondents responded to a forced-choice rank of seven potential improvements in bowel function. For each bowel function priority, the distribution of the rank scores that it received are shown.

in individuals with SCI causes infection, stones, bladder and kidney damage, etc. Respondents may be considering these challenges when reporting on their priorities. Catheterization of the bladder is usually required to void urine. Catheterization methods, such as indwelling or intermittent catheterization, can result in recurrent urinary tract infections that may require hospitalization [22]. Survey data suggested this same relationship, with most respondents using intermittent catheterization and most respondents also experiencing urinary tract infections, further supporting the need for solutions to empty the bladder without catheters and to reduce medical complications. Reducing or eliminating catheterization could therefore reduce urinary tract infections accordingly, though not necessarily solve all urinary tract infections.

\section{Bowel management}

The use of digital rectal stimulation with laxatives, suppositories, or mini-enemas were often reported. Almost half of respondents require at least some assistance with their 


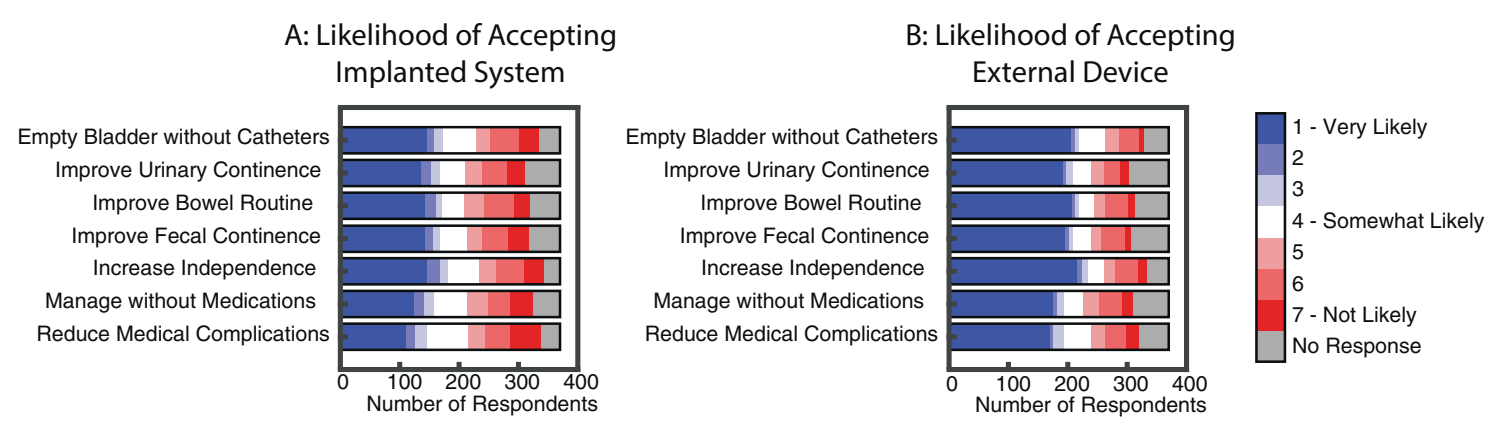

C: Ranked Concerns Associated with Implanted System

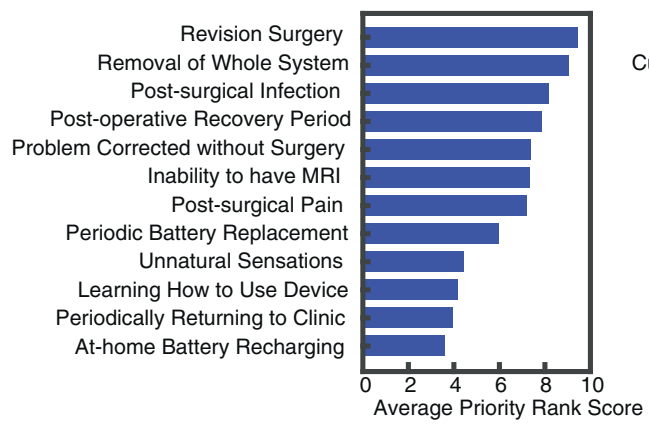

Fig. 3 Likelihood of accepting implanted or external neurostimulation systems. For each potential benefit, respondents rated their likelihood of adopting an implanted system (a) or an external system (b) on a scale from 1 to 7 , with 1 being "very likely" and 7 being "not likely". They provided this rating for each of seven potential functional benefits, including helping to empty their bladder without catheters; improving urinary continence; improving duration and predictability of the bowel routine; improving fecal continence; increasing independence in managing bladder or bowel function;

neurogenic bowel and about half of respondents are unable to sense when their bowel is full. These rates are higher than for bladder function, suggesting decreased independence for managing their bowel compared to their bladder. Over $60 \%$ of respondents required at least $30 \mathrm{~min}$ to empty their bowels and the rate of reported complications was high, including constipation and fecal incontinence. Respondents' neurogenic bowels also interfered with everyday activities. Bowel care duration, methods of management, and interference with quality of life are consistent with the literature [23-25]. These data demonstrate the adverse impact of neurogenic bowel dysfunction, the challenges and often dependence on others for managing it, and the lack of options for managing or improving bowel function after SCI.

Fecal continence; predictability in the bowel routine; and reduced bowel emptying time were the top ranked preferences for return of function. Most respondents experienced fecal incontinence, which can have a significant adverse impact on an individual's social life [26]. Indeed, most respondents reported that their bowel dysfunction

\section{D: Ranked Concerns Associated with External Device}

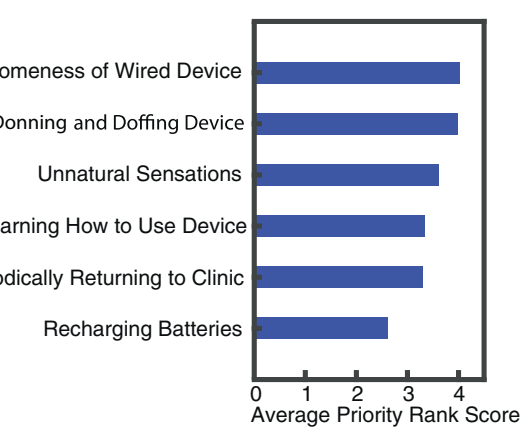

reducing the need for medications; and reducing medical complications associated with managing bladder or bowel function. Respondents rated their likelihood of accepting external or implanted devices consistently the same for these potential benefits, therefore, these scores were averaged across those seven potential benefits. Ranked concerns associated with implanted and external neurostimulation systems. For each potential risk, respondents made a forced rank choice, from most to least concerning, for potential risks associated with implanted systems (c) and external systems (d).

interfered with their work and social activities. Most respondents used digital rectal stimulation and/or manual evacuation in combination with laxatives, suppositories, or mini-enemas and still struggle with chronic constipation and long bowel emptying routines. Manual bowel programs may be required to eliminate feces, which can be timeconsuming and stigmatizing [23]. Only a third of respondents empty their bowels in under a half hour. Thus, the ranking of these bowel preferences is not unexpected.

\section{Attitudes toward neurostimulation approaches}

There was very little difference in how respondents rated the acceptability of nerve stimulation devices, given seven bladder or bowel potential benefits. Respondents demonstrated willingness to accept neurostimulation devices that would help address challenges associated with managing bladder and bowel function, such as urinary tract infections and fecal incontinence. This motivation is consistent with how respondents in another study evaluated existing methods for managing function following SCI [26]. There 
was a significant overall difference in how respondents rated external versus implanted devices. Respondents were more likely to adopt external devices than implanted devices, likely due to the different risk profiles. The largest concerns regarding external devices were regarding inconveniences of wearing an external system. The largest concerns for implanted devices involved complications from surgery. Nonetheless, a significant percentage of respondents were willing to try implanted neurostimulation devices to achieve these benefits. These results are consistent with preferences for neurostimulation approaches to restore bladder function as reported in another study [27]. Note that clinically relevant difference has not been defined for these metrics. The key take-away from this metric is that, overall, respondents were likely to very likely to accept these approaches. These results should be interpreted carefully, given that respondents were presented with an ideal solution wherein a hypothetical benefit was achieved absolutely. In practice, a neurostimulation device would provide a benefit with some rate of success or level of effectiveness that must be weighed against a rate and severity of risks that is particular to that device. Based on these results, we might expect a willingness to adopt these technologies, but study participants and consumers should be asked about how they rate this risk-benefit balance for a given neurostimulation device that they are testing.

\section{Study limitations}

It is difficult to evaluate the representativeness of these respondents to the SCI population due to different sources of bias and sampling limitations [28]. This Voice of Customer survey was disseminated and administered via internet, which may have missed disadvantaged individuals who lack internet access or are not well-connected to the SCI community. We sought responses from individuals with SCI, regardless of other circumstances, such as whether or not they had bladder or bowel dysfunction or how long they had an SCI, etc. Many people living with SCI are not connected to an SCI specialist. Therefore, this survey was disseminated by word-of-mouth by community influencers and social media, and by advertisement in newsletters of advocacy groups, which were all largely based in the United States and Canada. Though we provided an estimate of response rate, it is difficult to estimate the number of individuals that this survey reached and then, in turn, to estimate a response rate. This methodology limited the sample size and geographic representation, and there could be a self-selection bias among respondents who chose to complete the survey. However, there was large diversity among the respondents' experienced bladder and bowel dysfunctions and methods for managing these functions. As this study is modeled as a Voice of Customer study, we believe that the number of responses is sufficient to draw some helpful understanding about the trends for bladder and bowel priorities and attitudes toward neurostimulation approaches. Inclusion of respondents from other countries may affect some of the findings based on access to healthcare providers or different standards of practice, etc. Future studies should be conducted to address these limitations and include additional demographics and data from other international regions. Thus, we have captured the views and opinions of a select group of people and that without a clearly representative sample it is difficult to be sure how widespread these views are. Nonetheless, even knowing the views of some provides valuable insights.

We chose not to ask respondents about their American Spinal Injury Association Impairment Scale (AIS) grade or lesion level. This information would have helped us to evaluate how representative our sample is of the general SCI population, but this was not critical to the primary objectives of this study. This topic was discussed extensively with the Consumer Advisory Board during the survey design and there was a consensus among the Consumer Advisory Board members not to directly ask for AIS grades or lesion levels. They advised that AIS level does not translate directly to the language of those living with SCI since two individuals with the same AIS may function differently due to other confounding variables, and made the point that hand function and level of independence with bowel and bladder management is more important for this study. We asked specifically about hand function, level of independence, and bladder and bowel dysfunction. Our survey was not intended, therefore not designed, to be an outcome measure that other researchers would use to examine change over time, or diagnose or treat neurogenic bowel and bladder function. The questions were designed to explore people's perspectives and because there is no standardized questionnaire for this you have designed your own set of questions after careful consideration. It was designed by multiple scientists and multiple people with SCI, and tested independently by others with SCI. The survey was intended to inform researchers as they build new approaches that may have promise as a future treatment. The questions were designed to explore people's perspectives and to inform the development of emerging technology in this area.

Some of the co-authors who work with advocacy groups helped disseminate the survey, which could have influenced the respondents. To reduce this effect, co-authors were not named on the survey tool. Some of the co-authors have potential conflict of interest (see "Conflict of interest"), which could have influenced interpretation of results. To reduce this effect, we discussed results as a group, which included different stakeholders and the Consumer Advisory Board. 
Most of the respondents live in the United States and Canada, and data may be more reflective of a North American experience with SCI, but we do not have sufficient data to determine if location of residence is a factor in responses. The order of answer choices was not randomized, which may have influenced the ranking of choices in some questions. A specific nerve stimulation device was not presented, which will affect levels of risk and benefit. This questionnaire may be helpful for determining the acceptability of neurostimulation approaches in general, but individuals may evaluate the risks and benefits of particular devices differently. Therefore, more specific questionnaires with additional demographic data are needed for specific devices and approaches as they become available.

\section{Conclusion}

People living with SCI were queried regarding their bladder and bowel management strategies, priorities, and challenges, and on their attitudes toward potential neurostimulation approaches. Individuals who are more independent, with good hand function and less need for assistance in bladder or bowel emptying, prioritize technologies that would improve or restore bladder emptying without catheters and maintain urinary continence without medical complications. Individuals who are less independent may require additional technologies to improve their independence, such as improved hand function or trunk control for transfers and support during bladder and bowel emptying events. Most individuals may be willing to adopt neurostimulation approaches to achieve these bladder and bowel goals if those approaches meet their functional needs and are generally regarded as safe, but new surveys would be needed for specific approaches and their benefits and risks. The data from this survey could help guide the research and development of neurostimulation solutions specifically for this population.

\section{Data availability}

The datasets generated and analyzed during the current study are available from the corresponding author on reasonable request.

Funding This work was supported in part by the U.S. Department of Veterans Affairs Rehabilitation Research and Development Service RX001962. The contents do not represent the views of the U.S. Department of Veterans Affairs or the United States Government.

Author contributions DB, GC, BF, JF, TJ, AK, AR, BGS, KBT, TW, and JW contributed equally to survey design and implementation. DB, $\mathrm{AB}, \mathrm{WD}, \mathrm{RK}$, and JS contributed to data analyses and reporting.
All co-authors contributed to results interpretation and manuscript preparation.

\section{Compliance with ethical standards}

Conflict of interest KBT and BGS are co-authors and they work for Dignify Therapeutics Inc. and for Radiometer, respectively. These roles may present real or perceived conflict of interest.

Ethics We certify that all applicable institutional and governmental regulations concerning the ethical use of human volunteers were followed during the course of this research.

Publisher's note Springer Nature remains neutral with regard to jurisdictional claims in published maps and institutional affiliations.

\section{References}

1. Anderson KD. Targeting recovery: priorities of the spinal cordinjured population. J Neurotrauma. 2004;21:1371-83.

2. French JS, Anderson-Erisman KD, Sutter M. What do spinal cord injury consumers want? A review of spinal cord injury consumer priorities and neuroprosthesis from the 2008 neural interfaces conference. Neuromodulation J Int Neuromodulation Soc. 2010;13:229-31.

3. Bragge P, Piccenna L, Middleton JW, et al. Developing a spinal cord injury research strategy using a structured process of evidence review and stakeholder dialogue. Part I: rapid review of SCI prioritisation literature. Spinal Cord. 2015;53:714-20.

4. Braaf S, Lennox A, Nunn A, Gabbe B. Social activity and relationship changes experienced by people with bowel and bladder dysfunction following spinal cord injury. Spinal Cord. 2017;55:679-86.

5. Park SE, Elliott S, Noonan VK, et al. Impact of bladder, bowel and sexual dysfunction on health status of people with thoracolumbar spinal cord injuries living in the community. J Spinal Cord Med. 2017;40:548-59.

6. Ren J, Chew DJ, Biers S, Thiruchelvam N. Electrical nerve stimulation to promote micturition in spinal cord injury patients: a review of current attempts. Neurourol Urodyn. 2016;35:365-70.

7. Brindley GS. The first 500 patients with sacral anterior root stimulator implants: general description. Paraplegia. 1994;32:795-805.

8. Martens FMJ, Heesakkers JPFA. Clinical results of a brindley procedure: sacral anterior root stimulation in combination with a rhizotomy of the dorsal roots. Adv Urol. 2011;2011:709708.

9. Creasey GH. Electrical stimulation of sacral roots for micturition after spinal cord injury. Urol Clin North Am. 1993;20:505-15.

10. Wheeler JS, Walter JS, Sibley P. Management of incontinent SCI patients with penile stimulation: preliminary results. J Am Paraplegia Soc. 1994;17:55-9.

11. Lee Y-H, Creasey GH. Self-controlled dorsal penile nerve stimulation to inhibit bladder hyperreflexia in incomplete spinal cord injury: a case report. Arch Phys Med Rehabil. 2002;83:273-7.

12. Opisso E, Borau A, Rijkhoff NJM. Subject-controlled stimulation of dorsal genital nerve to treat neurogenic detrusor overactivity at home. Neurourol Urodyn. 2013;32:1004-9.

13. Bourbeau DJ, Gustafson KJ, Brose SW. At-home genital nerve stimulation for individuals with SCI and neurogenic detrusor overactivity: a pilot feasibility study. J Spinal Cord Med. 2019;42:360-70.

14. Bhadra N, Bhadra N, Kilgore K, Gustafson KJ. High frequency electrical conduction block of the pudendal nerve. J Neural Eng. 2006;3:180-7.

15. Craggs M, McFarlane J. Neuromodulation of the lower urinary tract. Exp Physiol. 1999;84:149-60. 
16. Wheeler TL, Bowel and Bladder Workshop Participants, de Groat W, Eisner K, Emmanuel A, French J, et al. Translating promising strategies for bowel and bladder management in spinal cord injury. Exp Neurol. 2018;306:169-76.

17. Anderson KD. Consideration of user priorities when developing neural prosthetics. J Neural Eng. 2009;6:055003.

18. Myer ENB, Petrikovets A, Slocum PD, et al. Risk factors for explantation due to infection after sacral neuromodulation: a multicenter retrospective case-control study. Am J Obstet Gynecol. 2018;219:78.e1-e9.

19. Weber PB, Kapur R, Gwinn RP, Zimmerman RS, Courtney TA, Morrell MJ. Infection and erosion rates in trials of a cranially implanted neurostimulator do not increase with subsequent neurostimulator placements. Stereotact Funct Neurosurg. 2017;95:325-9.

20. Wexner SD, Hull T, Edden $\mathrm{Y}$, et al. Infection rates in a large investigational trial of sacral nerve stimulation for fecal incontinence. J Gastrointest Surg J Soc Surg Aliment Tract. 2010;14:1081-9.

21. National Spinal Cord Injury Statistical Center. Spinal cord injury facts and figures at a glance. J Spinal Cord Med. 2014;37:355-6.

22. Wyndaele J-J. The management of neurogenic lower urinary tract dysfunction after spinal cord injury. Nat Rev Urol. 2016;13:705-14.
23. Inskip JA, Lucci V-EM, McGrath MS, Willms R, Claydon VE. A community perspective on bowel management and quality of life after spinal cord injury: the influence of autonomic dysreflexia. J Neurotrauma. 2018;35:1091-105.

24. Adriaansen JJ, van Asbeck FW, van Kuppevelt D, Snoek GJ, Post MW. Outcomes of neurogenic bowel management in individuals living with a spinal cord injury for at least 10 years. Arch Phys Med Rehabil. 2015;96:905-12.

25. Burns AS, St-Germain D, Connolly M, et al. Phenomenological study of neurogenic bowel from the perspective of individuals living with spinal cord injury. Arch Phys Med Rehabil. 2015;96:49-55.

26. Nafees B, Lloyd AJ, Ballinger RS, Emmanuel A. Managing neurogenic bowel dysfunction: what do patients prefer? A discrete choice experiment of patient preferences for transanal irrigation and standard bowel management. Patient Prefer Adherence. 2016;10:195-204.

27. Sanders PMH, Ijzerman MJ, Roach MJ, Gustafson KJ. Patient preferences for next generation neural prostheses to restore bladder function. Spinal Cord. 2011;49:113-9.

28. Harvey LA, Dijkers MP. Surveys can provide valuable data but careful consideration needs to be given to the methods used to sample the population of interest. Spinal Cord. 2020;58:257-8. 